\title{
Uso de métodos Monte Carlo na modelagem de fontes radioativas naturais
}

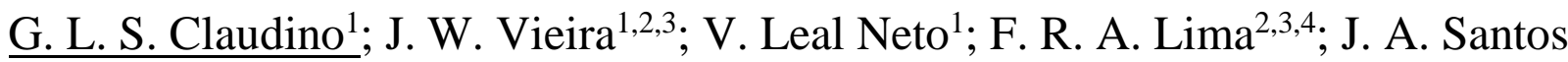 \\ Junior $^{3}$; R. S. Amaral ${ }^{3}$ \\ ${ }^{1}$ Instituto Federal de Educação, Ciência e Tecnologia de Pernambuco, IFPE Campus Recife \\ CEP 50740-540, Av. Prof. Luís Freire, 500, Recife-PE, Brasil \\ ${ }^{2}$ Escola Politécnica de Pernambuco, EPP-UPE \\ CEP 50750-470, Rua Benfica, 455, Recife-PE, Brasil \\ ${ }^{3}$ Departamento de Energia Nuclear, DEN-UFPE \\ CEP 50740-540, Av. Prof. Luís Freire, 1000, Recife-PE, Brasil \\ ${ }^{4}$ Centro Regional de Ciências Nucleares do Nordeste, CRCN-NE/CNEN \\ CEP 50740-540, Av. Professor Luiz Freire, 200, Recife-PE, Brasil \\ E-mail: berg2020@hotmail.com
}

\begin{abstract}
Exist, in Brazil and in the world, different places with high levels of ambient radiation, mainly due to the presence of primordial radionuclides, exposing populations to risks that can not be neglected. To evaluate the distribution of the absorbed dose to organs and tissues of a human body, the Group of Numerical Dosimetry (GDN) utilizes Computational Models Exhibition (MCES), that use a voxel phantom and the Monte Carlo code EGSnrc to simulate the transport of radiation, its interaction with the atoms of the medium and evaluate the energy deposited in regions of interest. Essentially, if tried improve the way the photons are sampled in the plan, taking in account the probability density function (PDF) that describes the problem, the runtime simulation and dosimetric results. This article, was simulated a circular region of soil with the phantom center and limited by a maximum radius $\left(r_{\text {max }}\right)$, from where, because of concentration of natural radioactive elements, photons can emerge with diverse energies and radial distribution similar to that modeled by GDN. The objective is to investigate the influence of $r_{\max }$ in the dosimetric results. To perform the simulations was adapted MCE MSTA, composed by phantom MASH (Male Adult meSH) in orthostatic position coupled to EGSnrc, with the algorithm of FDP NT allowing execution for different values of $r_{\max }$. From $r_{\max }=2000 \mathrm{~cm}$, It is found that the dosimetric values do not vary significantly, no offsetting the increased computational time spent to simulate higher values of $r_{\max }$.
\end{abstract}

Keywords: Natural Radiation, Exposure Computational Models, Planar Source 


\section{INTRODUÇÃO}

Para estimar a distribuição de dose absorvida pelos órgãos e tecidos radiossensíveis de pessoas submetidas às radiações ionizantes, é necessário realizar simulações utilizando um MCE. Tais modelos são compostos, fundamentalmente, por um simulador antropomórfico (fantoma, neologismo da palavra inglesa phantom), um código MC e um algoritmo da fonte radioativa (VIEIRA, 2004).

O GDN tem contribuído significativamente para o desenvolvimento e utilização de fantomas de voxels (VOlume piXELS), desde o MAX (Male Adult voXel) (KRAMER el al., 2003) até MASH (Male Adult meSH) (CASSOLA, 2011). Os códigos MC estão bem estabelecidos na comunidade científica e o GDN tem utilizado o sistema EGS (Electron Gamma Shower), principalmente o EGSnrc (KAWRAKOW et al., 2013) para simular o transporte de fótons e elétrons através dos materiais constituintes do fantoma, as interações destes com os átomos do meio e a avaliação da energia depositada em regiões de interesse. O grupo também tem desenvolvido algoritmos simuladores de fontes radioativas emissoras de fótons como os apresentados em Vieira (2004).

O GDN desenvolveu um algoritmo de uma fonte planar para simular a radiação gama natural advinda de uma porção plana do solo que pode atingir uma pessoa em posição ortostática. Considerando que o problema tem simetria azimutal ( $\theta$ é uma variável aleatória uniforme em [0, 2 $\pi]$ ), Kramer, (1979), deduziu a função densidade de probabilidade (FDP) para a variável radial $r$, modelando o plano como um círculo de raio $r_{\max }$. Integrando esta FDP é possível obter a fórmula analítica da função de distribuição acumulada (FDA) do problema, mas esta não é inversível, o que inviabiliza a obtenção de um gerador de números aleatórios (GNA) radiais por via direta. Em 2006, Leal Neto e colaboradores apresentaram um GNA para $r$ baseado em amostragens por técnicas MC de rejeição com uma FDP exponencial por sobre o perfil da FDP do problema. Em 2012, Vieira e colaboradores desenvolveram um algoritmo baseado na FDP de Johnson SD (JOHNSON, 1949), renomeada nas publicações do GDN para FDP Normal Transladada (NT), que se mostrou o mais preciso e eficiente até então. Os resultados dosimétricos obtidos com este algoritmo NT foram comparados com similares obtidos por Leal Neto e colaboradores (2006).

Neste trabalho, é apresentado um novo estudo sobre fonte de radiação natural planar: a otimização do $r_{\max }$ em termos dosimétricos e computacionais. O estudo consistiu em investigar o comportamento do $r_{\max }$, a partir das grandezas de saída das simulações MC utilizando o GNA_NT: o tempo computacional e os valores estimados para o coeficiente de conversão E/INAK, onde $E$ é a dose efetiva e INAK (INcident Air Kerma) é a grandeza de normalização KERMA (Kinetic Energy Released per unit MAss) incidente no ar. Os fótons simulados podem ter origem nos elementos radioativos naturais Urânio-238, Torio-232, Radio-226 ou Potássio-40 (IAEA, 2003; RAMASANY et al., 2013), e emergir para uma região superior do solo. A frequência com que um dado tipo de fóton é sorteado foi baseada em concentrações dos elementos e suas energias: U-238, com 863,31 Bq/kg de solo, e energias de 13 e $40 \mathrm{keV}$; Th-232, 
com 719,54 Bq/Kg de solo, e energia de $59 \mathrm{keV}$; Ra-226, com 612,56 Bq/kg de solo, e energias de 81, 83, 94, 97, 185, 262, 414, 449 e $600 \mathrm{keV}$; e o K-40, com 539,53 Bq/kg de solo e energia de $1460 \mathrm{keV}$. Estes dados foram introduzidos no algoritmo da fonte que retorna uma das energias possíveis a cada chamada no código do MCE.

Para fins da proteção radiológica, os valores anuais de $E$ não devem ultrapassar limites estabelecidos por entidades regulamentadoras como a CNEN (Comissão Nacional de Energia Nuclear) e a ICRP (International Commission on Radiological Protection, Comissão Internacional de Proteção Radiológica). Assim, obtendo os coeficientes E/INAK e medindo INAK em um tempo $t$, nas mesmas condições simuladas, pode-se avaliar a taxa de dose efetiva no fantoma causada pela fonte planar. Esta taxa pode ser extrapolada para um ano e comparada com limites estabelecidos. A melhoria do algoritmo gerador de $r$ nos MCEs utilizados reflete em avaliações dosimétricas mais exatas, fundamentais para a proteção radiológica de trabalhadores e pessoas do público.

As implementações necessárias, tanto na preparação de arquivos de entrada em formatos adequados para leitura pelo EGSnrc, quanto na análise gráfica e numérica dos dados gerados nas simulações MC, foram realizadas no ambiente de desenvolvimento integrado do Microsoft Visual Studio 2013 (MVS 2013), usando a linguagem de programação C\# e a de marcação XAML no tipo de projeto chamado WPF Application (XU, 2009). A Figura 1a mostra a janela principal do software Análise Monte Carlo (AMC) (CLAUDINO et al., 2013), destacando o menu Arquivos, onde foram alocadas as implementações para arquivos de entrada; A Figura 1b destaca os itens de menu implementados especialmente para este trabalho.

Figura 1 - Janela principal do software AMC exibindo (a) o conteúdo do menu Arquivos e (b) o conteúdo do menu Análise Gráfica e Numérica.

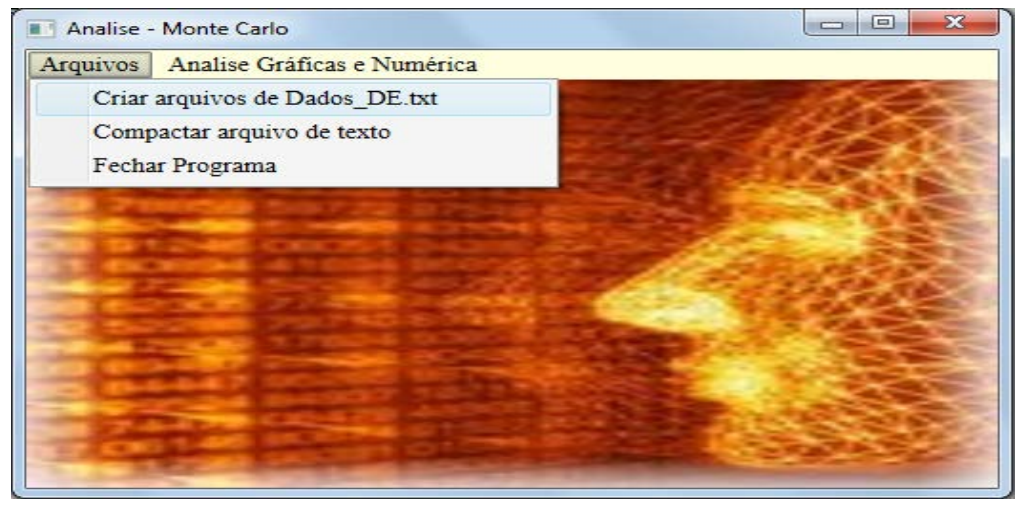




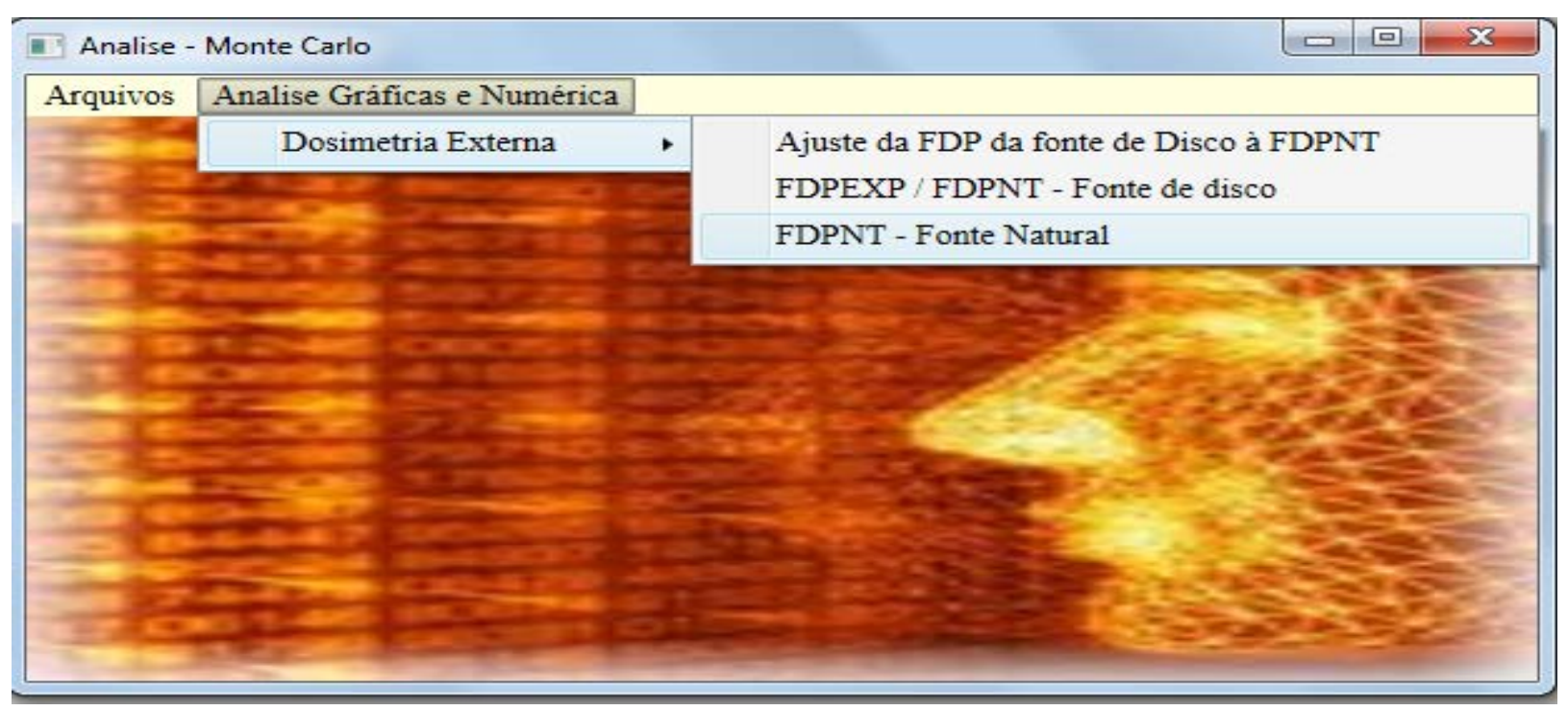

\section{MATERIAIS E MÉTODOS}

Este trabalho foi desenvolvido no Laboratório de Dosimetria Numérica do Instituto Federal de Educação, Ciência e Tecnologia de Pernambuco (IFPE), Campus Recife, em computadores que têm como principais itens de configuração um processador Intel(R) Core(TM) i7 CPU X990 @ 3,47GHz, 24 GB de RAM e o sistema operacional Windows 7 Ultimate de 64 bits. Nestes computadores estão instalados os softwares aqui utilizados: o EGSnrc, o MVS 2013 Ultimate para 32 bits, o Excel 2007 e o AMC. O fantoma MASH foi obtido em http://www.caldose.org/, bem como os arquivos originais que compõem o MCE MSTA. A interface para análise gráfica e numérica dos resultados dosimétricos, cujo caminho de acesso no AMC é mostrado na Figura 1b, foi construída com o auxílio da versão livre da biblioteca de vínculo dinâmico AmCharts.Windows.dll (nos gráficos apresentados nos resultados há um hiperlink para a página web de onde foi obtida a versão usada).

A Figura 2 ilustra a situação-problema modelada (LEAL NETO et al., 2006): fótons dos elementos radioativos naturais que emergem, isotropicamente, de uma região circular e podem atingir uma pessoa em pé no centro do círculo.

Figura 2: Ilustração da situação-problema (LEAL NETO et al., 2006).

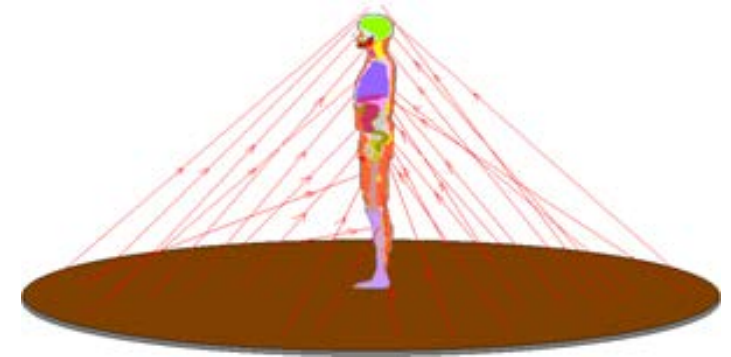




\section{A FDP do Problema}

A dedução matemática da FDP radial, iniciada em Kramer (1970) e concluída em Leal Neto colaboradores (2006), consiste em considerar uma área preenchida uniformemente com fontes pontuais que, isotropicamente, emitem fótons no espaço $2 \pi$ superior. Dependendo do modo de propagação do material radioativo e da constituição do meio, fontes deste tipo podem ter formas e tamanhos bem diferentes. Mas, mesmo se o tamanho de uma região circular considerada tender a infinito, por exemplo, para simular a radiação natural terrestre, é razoável supor que existe um raio limiar, $r=r_{\max }$, de tal maneira que uma pessoa em pé não seria atingida pela radiação emergente da área onde $r>r_{\max }$ de modo significativo. A Figura 3 mostra um esboço da geometria a ser usada para deduzir uma expressão matemática para a distribuição de fótons em uma superfície cilíndrica envolvendo o fantoma irradiado.

Figura 3: Esboço da geometria da fonte planar.

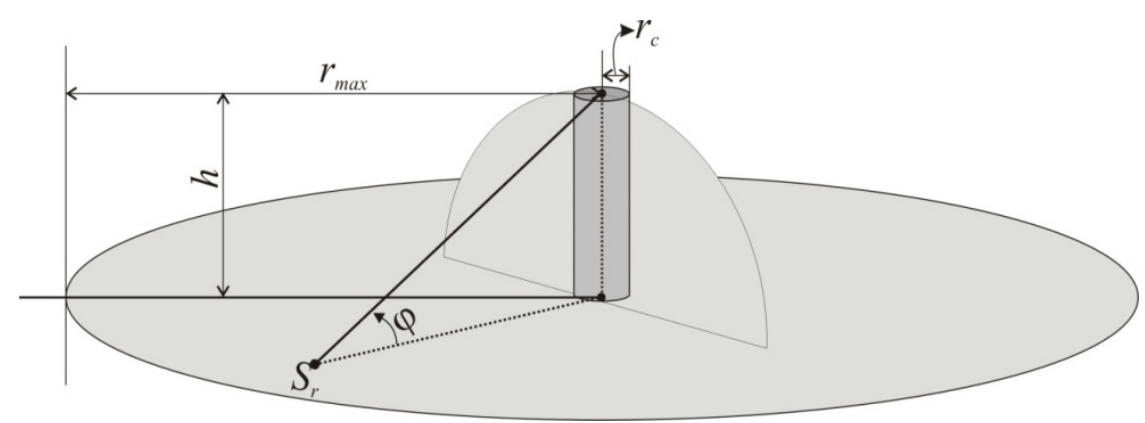

O GNA pretendido deve sortear fótons emergentes de dentro de um círculo de raio $r_{\max } \mathrm{e}$ fora de um círculo de raio $r_{c}$, que atinjam a superfície cilíndrica de altura $h$ e raio $r_{c}$. Na região com $r<r_{c}, r$ é uniformemente amostrado no intervalo [0, $r_{c}$ ]. Se a distribuição dos fótons que chegam ao semicírculo de raio $h$ da Figura 3 em função da distância $r$ for conhecida, pode-se, a partir do ponto $S r$ da figura, obter a FDP radial que descreve a fonte planar. Cada fóton emergente do ponto $S r$, obtido a partir de um $r$ dependente da FDP a ser deduzida e de um ângulo $\theta$, uniformemente distribuído em $[0,2 \pi]$, poderá atingir a área lateral do cilindro de raio $r_{c}$. Caso não atinja será rejeitado e novo ponto $S_{r}$ será sorteado. Mesmo os fótons que atinjam a área cilíndrica poderão ser rejeitados caso não entrem no fantoma.

A Figura 4 mostra o esquema para um fóton útil, que emerge de $\mathrm{Sr}$ e voa sob um ângulo 0 $\leq \varphi(r)<\pi / 2$. Para descrever matematicamente esta situação, o círculo foi separado nas regiões 0 $\leq r \leq r_{c}$ e $r_{C}<r \leq r_{\max }$. 
Figura 4: Variáveis usadas na dedução da fórmula da FDP do problema.

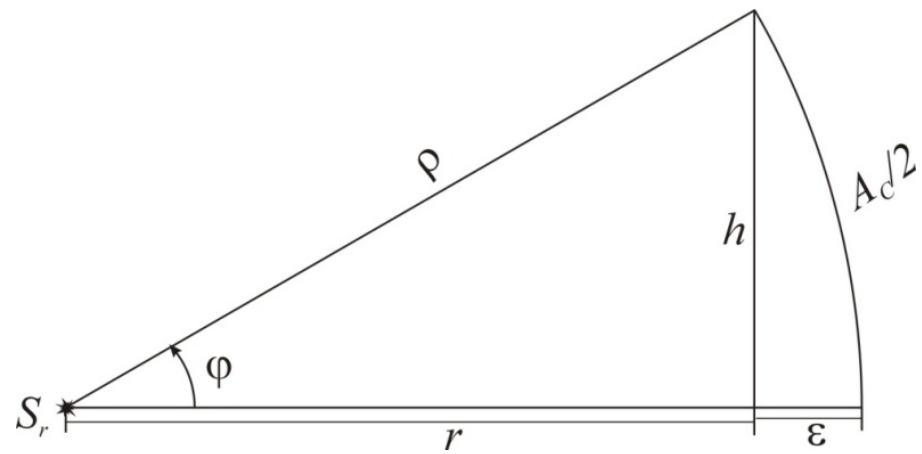

Em $0 \leq r \leq r_{c}$, região sob o fantoma,

$$
\left\{\begin{array}{l}
\theta=2 \pi \cdot x_{1} \\
r=r_{c} \cdot x_{2}
\end{array},\right.
$$

onde $x_{1}$ e $x_{2}$ são variáveis aleatórias uniformemente distribuídas em [0,1]. Resta deduzir a FDP a ser usada em $r_{c}<r \leq r_{\max }$.

A área da calota cuja metade do seu contorno é mostrada na Figura 4 pode ser calculada usando a proporção $\frac{A_{C}}{4 \pi \rho^{2}}=\frac{\varepsilon}{2 \rho}$.

Isolando $A_{C}: A_{C}=2 \pi \rho \varepsilon=2 \pi \rho\left(\rho-\sqrt{\rho^{2}-h^{2}}\right) \Rightarrow$

$$
A_{C}=2 \pi \rho^{2}\left(1-\frac{\sqrt{\rho^{2}-h^{2}}}{\rho}\right) .
$$

Da Figura 4:

$$
\cos \varphi(r)=\frac{\sqrt{\rho^{2}-h^{2}}}{\rho}=\frac{r}{\sqrt{r^{2}+h^{2}}} .
$$

Substituindo (3) em (2):

$$
A_{C}(r)=2 \pi\left(r^{2}+h^{2}\right)[1-\cos \varphi(r)] .
$$

O ângulo sólido da calota completa é: $\Omega(r)=\frac{A_{C}}{r^{2}+h^{2}}=2 \pi[1-\cos \varphi(r)]$.

Para a meia calota mostrada na Figura 4:

$$
\Omega_{m c}(r)=\pi[1-\cos \varphi(r)] .
$$


Fótons que emergem de uma fonte pontual em $\mathrm{Sr}$ para o semiespaço acima do círculo, voam sob um ângulo sólido total de $\Omega_{\text {total }}=2 \pi$ srad. Assim, a probabilidade $W(r)$ de um fóton sair de $S r$ e atingir a meia calota da Figura 4 é

$$
W(r)=\frac{\Omega_{m c}(r)}{\Omega_{\text {total }}}=\frac{1}{2}[1-\cos \varphi(r)] .
$$

O número de fótons que emerge com isotropia azimutal de um anel com largura $d r$ é

$$
d N_{\text {iso }}=\frac{2 \pi r d r}{\pi r_{\max }^{2}} N=\frac{2 r d r}{r_{\max }^{2}} N,
$$

onde $N$ é o número total de fótons simulados. Consequentemente o número de fótons que sai do anel circular no plano e chega à meia calota é, usando (5) e (6):

$$
\begin{aligned}
d N=d N_{i s o} W(r)=\frac{2 r d r}{r_{\max }^{2}} N \frac{[1-\cos \varphi(r)]}{2} & \Rightarrow \\
d N & =N \frac{r}{r_{\max }^{2}}[1-\cos (\varphi(r))] d r .
\end{aligned}
$$

Assim, a FDA, que dá a probabilidade de que fótons emergentes da região $r_{c}<r \leq r_{\max }$ atinjam a meia calota da Figura 4, é dada por:

$$
F(r)=\frac{\int_{r_{c}}^{r} r^{\prime}\left[1-\cos \varphi\left(r^{\prime}\right)\right] d r^{\prime}}{\int_{r_{c}}^{r_{\max }} r[1-\cos \varphi(r)] d r}=\frac{\int_{r_{c}}^{r}\left(r^{\prime}-\frac{r^{\prime 2}}{\sqrt{r^{\prime 2}+h^{2}}}\right) d r^{\prime}}{\int_{r_{c}}^{r_{\max }}\left(r-\frac{r^{2}}{\sqrt{r^{2}+h^{2}}}\right) d r} .
$$

Com algum esforço, as integrais acima podem ser resolvidas e obtém-se:

$$
F(r)=\frac{1}{C_{\max }-C_{\min }}\left[r^{2}-r \sqrt{r^{2}+h^{2}}+h^{2} \ln \left(r+\sqrt{r^{2}+h^{2}}\right)-C_{\min }\right],
$$

onde

$$
\left\{\begin{array}{l}
C_{\min }=r_{c}^{2}-r_{c} \sqrt{r_{c}^{2}+h^{2}}+h^{2} \ln \left(r_{c}+\sqrt{r_{c}^{2}+h^{2}}\right) \\
C_{\max }=r_{\max }^{2}-r_{\max } \sqrt{r_{\max }^{2}+h^{2}}+h^{2} \ln \left(r_{\text {max }}+\sqrt{r_{\text {max }}^{2}+h^{2}}\right)
\end{array} .\right.
$$

A FDP correspondente é determinada por $f(r)=\frac{d F(r)}{d r}$ :

$$
f(r)=\frac{2}{C_{\max }-C_{\min }}\left(r-\frac{r^{2}}{\sqrt{r^{2}+h^{2}}}\right)=A\left(r-\frac{r^{2}}{\sqrt{r^{2}+h^{2}}}\right) \text {. }
$$


A Figura 5 mostra os perfis de $F(r)$ e $f(r)$ usando os dados da Tabela 1. Figura 5: Perfis da FDA e da FDP do problema.
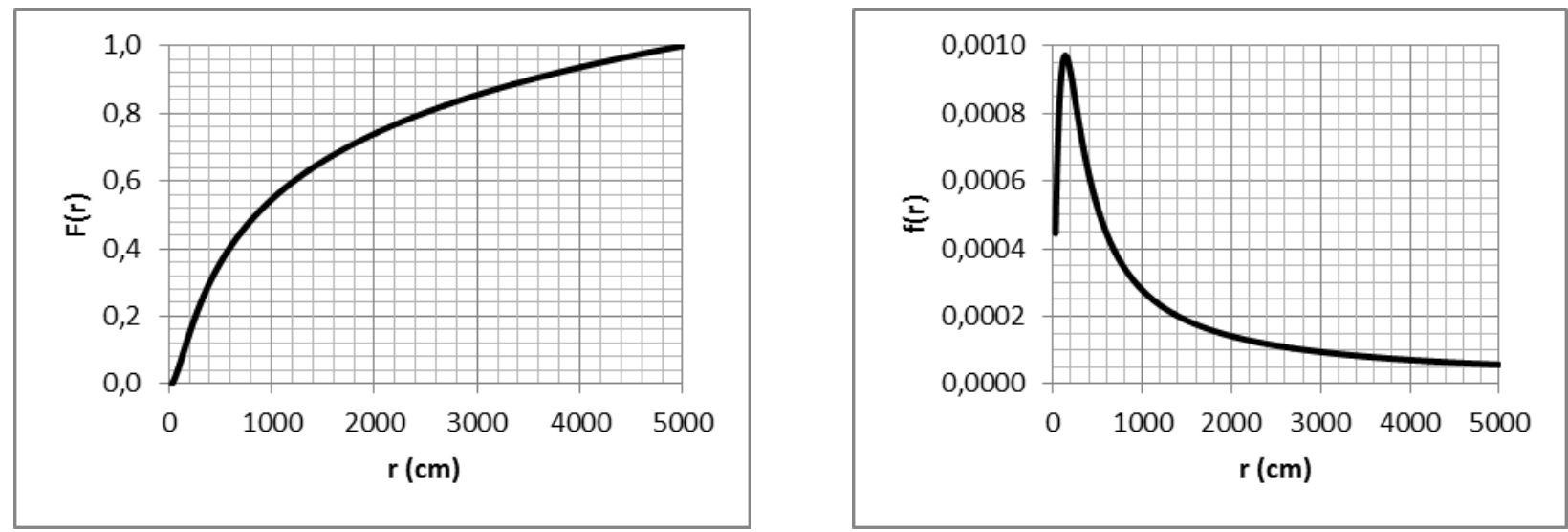

Tabela 1: Parâmetros da fonte planar para o MCE MSTA.

\begin{tabular}{cc}
\hline Constante & Valor \\
\hline$r_{c}(\mathrm{~cm})$ & 28,80 \\
$r_{\max }(\mathrm{cm})$ & 5000,00 \\
$h(\mathrm{~cm})$ & 175,56 \\
$A\left(\mathrm{~cm}^{-2}\right)$ & $1,84408 \mathrm{E}-05$ \\
\hline
\end{tabular}

\section{Porque não é Possível Obter um GNA Direto}

Na FDP do problema, (11), $h$ é a altura do fantoma MASH e $A$ é uma constante de normalização que depende de $h$, de $r_{c}$ e de $r_{\max }$. A FDA correspondente, (9), não é inversível o que inviabiliza a amostragem de $r$ por técnicas MC diretas como demonstrado a seguir.

Para distribuições univariantes, há uma técnica de inversão generalizada que pode ser usada para obtenção de um GNA transformado a partir da FDA uniforme (KALOS e WHITLOCK, 1986). Supondo que $x$ seja uma variável aleatória com FDA $F(x)$ e FDP obtida por $f(x)=d F(x) / d x$, e que $r=r(x)$ seja uma função contínua não decrescente de $x$, o que significaria a função $F(r)$ ? Com base na suposição acima, uma propriedade geral entre a variável $x$ e a função $r(x)$ pode ser expressa por

$$
r(X) \leq r(x) \text { se } X \leq x .
$$

Consequentemente, tem-se para as probabilidades,

$$
P\{r(X) \leq r(x)\}=P\{X \leq x\},
$$

ou, para as FDAs, 


$$
\left\{\begin{array}{l}
F(r)=F(x) \\
r=r(x)
\end{array} .\right.
$$

A relação entre as FDPs pode ser determinada diferenciando (14):

$\frac{d F(r)}{d x}=\frac{d F(x)}{d x} \Rightarrow \frac{d F(r)}{d r} \frac{d r}{d x}=f(x) \Rightarrow$

$$
f(r) \frac{d r}{d x}=f(x) .
$$

Como probabilidades são números positivos,

$$
f(r)=f(x)\left|\frac{d r}{d x}\right|^{-1}
$$

Se $x$ é uma variável uniforme, sua FDA é

$$
F(x)=\left\{\begin{array}{l}
0, x<0 \\
x, 0 \leq x \leq 1 \\
1, x>1
\end{array}\right.
$$

Portanto, o GNA $r=r(x)$ é determinado pela solução da equação

$$
F(r)=\int_{-\infty}^{r} f\left(r^{\prime}\right) d r^{\prime}=x
$$

Usando em (18) a FDA do problema:

$$
\frac{1}{C_{\max }-C_{\min }}\left[r^{2}-r \sqrt{r^{2}+h^{2}}+h^{2} \ln \left(r+\sqrt{r^{2}+h^{2}}\right)-C_{\min }\right]=x ! ! !
$$

Da equação acima se vê que é praticamente impossível obter diretamente um GNA radiais, o que levou o GDN a procurar métodos alternativos para amostrar $r$ e otimizar o algoritmo da fonte radioativa planar.

\section{Substituição da FDP Problema}

O novo algoritmo simulador da fonte planar é derivada da FDP NT,

$$
f(r)=\frac{\delta}{\sqrt{2 \pi}} \frac{1}{(1-r) r} e^{-\frac{1}{2}\left[\gamma+\ln \left(\frac{r}{1-r}\right)\right]^{2}}
$$


foi originalmente apresentado por Johnson (1949). A equação (20) mostra esse algoritmo em função da variável aleatória gaussiana padrão $z$ e dos parâmetros reais $\delta$ e $\gamma$ (VIEIRA et al., 2012):

$$
r=\frac{1}{1+e^{-\frac{(z-\gamma)}{\delta}}}
$$

Portanto o MCE MSTA_NT montado é composto pelo código EGSnrc, o fantoma MASH [CASSOLA, 2011] e o algoritmo da equação (20). O mesmo foi utilizado neste trabalho para realizar as simulações. Os resultados obtidos foram integrados ao software AMC.

\section{RESULTADOS E DISCUSSÕES}

O MSTA_NT foi executado com N (número de histórias) de 8E7 (CLAUDINO et al., 2013), sendo emito, para cada $\mathrm{N}$, um fóton energia dos elementos naturais já citados. Os dados obtidos foram adicionados ao arquivo de texto para dosimetria externa do software AMC. Os coeficientes de conversão escolhidos para analisar os resultados dosimétricos foram D/INAK e E/INAK. Como o fantoma usado é o MASH, E é a dose efetiva masculina. O tempo computacional foi calculado no AMC a partir das strings impressas nos arquivos de saída para início e fim de uma dada simulação. Estas strings estão no formato dia-mês-ano e hora:minuto:segundo.

\section{Otimização do Tamanho do Raio Máximo}

Nas simulações com o MCE MSTA_NT foram usados, em $\mathrm{cm}, r_{\max }=30,80,130,180,230,280$, $330,380,430,480,530,580,630,680,730,780,830,880,930,980,1000,1500,2000,2500,3000$, 3500, 4000, 4500, 5000, 5500, 6000, 6500, 7000, 7500, 8000, 8500, 9000, 9500 e 10000. As Figuras 6 e 7 foram obtidas a partir dos resultados de saída. 
Figura 6: Resultados dosimétricos do MSTA_NT.
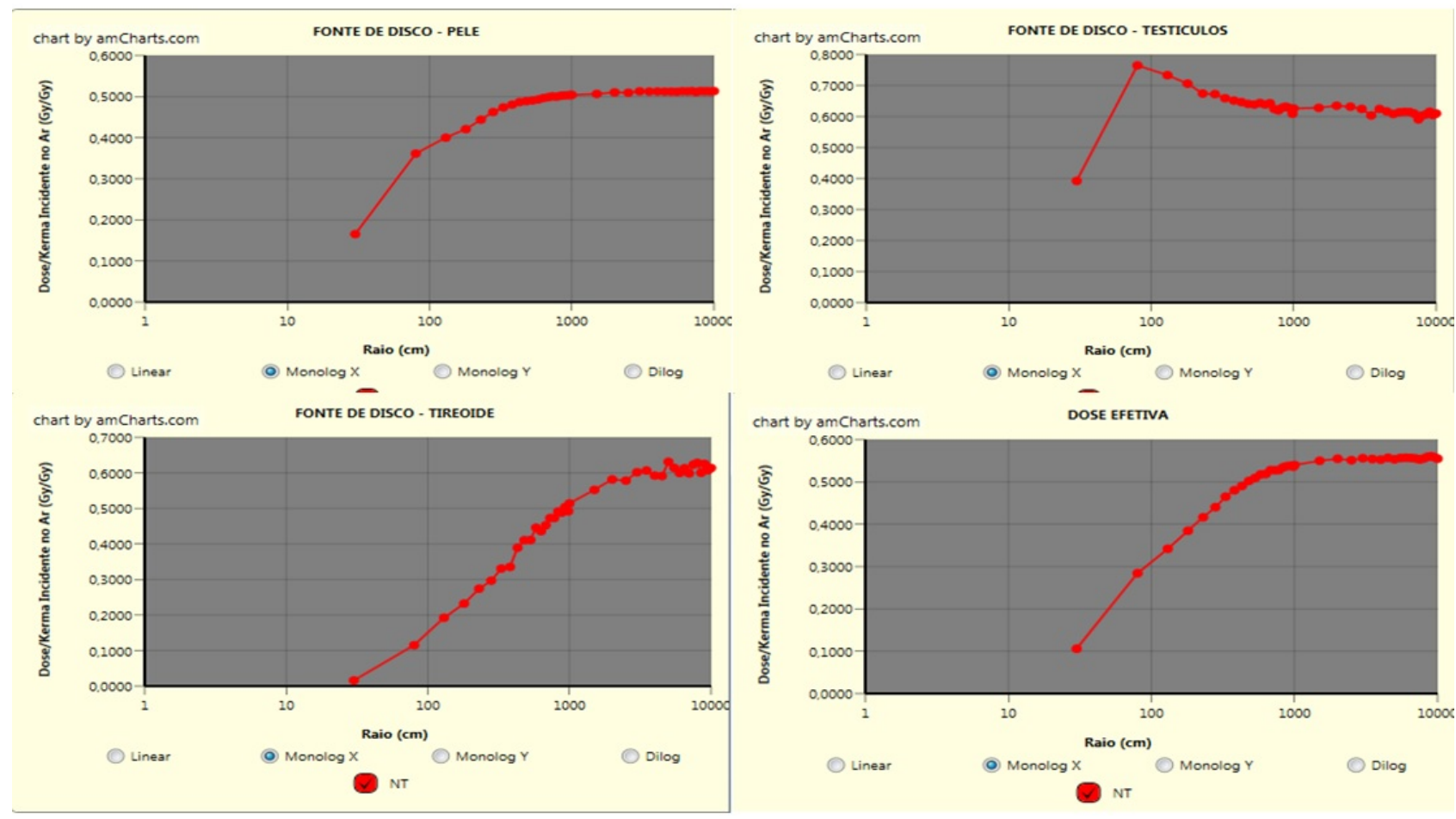

Figura 7: Resultado do Tempo Computacional para cada raio do MSTA_NT.

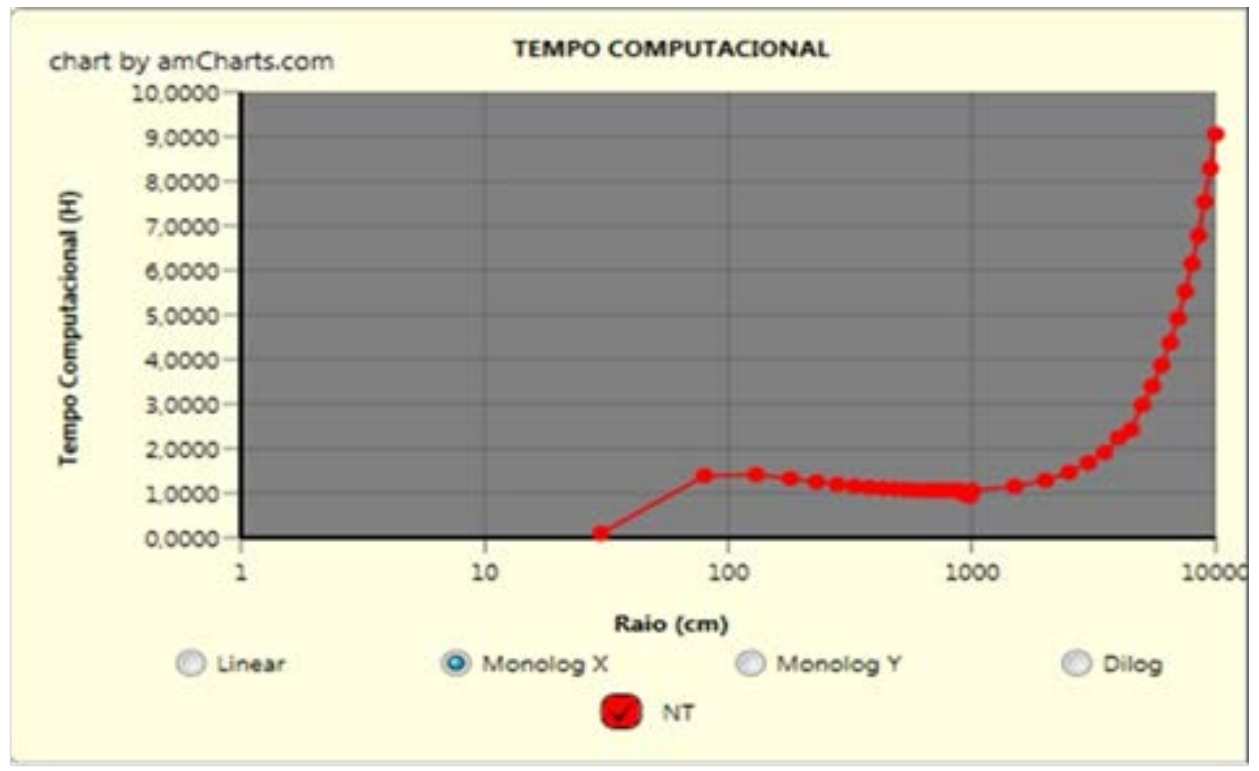

Como a simulação é de um plano infinito, se espera que os valores de E/INAK mais exatos sejam os obtidos com o maior $r_{\max }$ possível. Pode-se vê no último gráfico da Figura6 que os valores dosimétricos praticamente não variam a partir de $2000 \mathrm{~cm}$ para as energias simuladas. Assim, escolheuse $r_{\max }=2000 \mathrm{~cm}$ como um valor de raio que não afeta as estimativas dosimétricas e melhora 
substancialmente o tempo computacional em relação ao máximo valor de $r_{\max }$ simulado, como se vê na Figura 7.

\section{CONCLUSÕES}

Os gráficos das Figuras 6 e 7, bem como os valores de $E$ (ou D)/INAK e do tempo computacional tabulados em função dos 39 valores de $r_{\max }$ nas planilhas do software AMC, acessadas via os dois caminhos mostrados na Figura $1 \mathrm{~b}$, permitiram estabelecer o valor do $r_{\max }$ para $2000 \mathrm{~cm}$. Assim, 0 algoritmo simulador de fonte planar acoplado ao EGSnrc a ser utilizado nos futuros trabalhos do GDN conterá este valor otimizado.

\section{AGRADECIMENTO}

Os autores agradecem ao IFPE, ao CRCN-NE, à FACEPE e ao CNPq pelo apoio financeiro.

\section{REFERÊNCIAS}

1. CASSOLA, V. F.; Desenvolvimento de Fantomas Humanos Computacionais Usando Malhas Poligonais em Função da Postura, Massa e Altura, Tese Doutorado em Tecnologias Energéticas Nucleares, DEN-UFPE, Recife-PE, Brasil, 2011.

2. CLAUDINO, G. L. S.; VIEIRA, J. W.; LEAL NETO, V.; LIMA, F. R. A., Desenvolvimento de um algoritmo simulador de fonte radioativa planar para avaliações dosimétricas em acidentes envolvendo radiofármacos usados em medicina nuclear, CIENTEC, 5, № 2, 24-31, 2013.

3. IAEA, Guidelines for Radioelment Mapping Using Gamma Ray Spectrometry Data, International Atomic Energy Agency, IAEA-TECDOC-1363, Vienna, 2003.

4. JOHNSON, N. L., Systems of Frequency Curves Generated by Methods of Translation, Biometrika, 36, No. 1/2, 149-176, 1949.

5. KALOS, M. H.; WHITLOCK, P. A., Monte Carlo Methods, John Wiley \&Sons, Inc, 1986.

6. KAWRAKOW, I.; MAINEGRA-HING, E.; ROGERS, D. W. O. TESSIER, F.; WALTERS, B. R. B., The EGSnrc Code System: Monte Carlo Simulation of Electron and Photon Transport, NRCC Report PIRS-701, National Research Council of Canada, Ottawa, Canada, 2013.

7. KRAMER R.; Ermittlung von Konversionsfaktoren Zwischen Koerperdosen und Relevanten Strahlungskenngroessen bei Externer Roentgen-und Gamma-Bestrahlung, Gesellschaft fuer Strahlen-und Umweltforschung, Muenchen-Neuherberg, GSF-Bericht-S-556, 1979.

8. KRAMER, R.; VIEIRA, J. W.; KHOURY, H. J.; LIMA, F. R. A.; FUELLE, D., All About Max: A Male Adult Voxel Phantom for Monte Carlo Calculations in the Area of Radiation Protection Dosimetry, Phys. Med. Biol., 48, 1239-1262, 2003. 
9. LEAL NETO, V.; VEIRA, J. W.; STOSIC B.; LIMA, F. R. A., Desenvolvimento Computacional de uma Fonte Radioativa Isotrópica no Solo Utilizando Técnicas Monte Carlo de Amostragem por Rejeição, Primeiro Congresso Americano do IRPA 2006, XXIV Reunião Anual da SMSR e XVII Congresso Anual da SNM, Acapulco, México, de 3 a 8 de setembro, 2006.

10. RAMASAMY, V.; SUNDARRAJAN, M.; PARAMASIVAM, K.; MEENAKSHISUNDARAM, V.; SURESH, G., Assessment of spatial distribution and radiological hazardous nature of radionuclides in high background radiation area, Kerala, India, ELSEVIER, Applied Radiation and Isotopes, 73, 21 31, 2013.

11. VIEIRA, J. W., Construção de um Modelo Computacional de Exposição para Cálculos Dosimétricos Utilizando o Código Monte Carlo EGS4 e Fantomas de Voxels, Tese de Doutorado em Tecnologias Energéticas Nucleares, DEN-UFPE, Recife-PE, Brasil, 2004.

12. VIEIRA, J. W.; LEAL NETO, V.; LIMA FILHO, J. M.; LIMA, L. F.; LOPES FILHO, F. J.; ROCHA, E. A.; LIMA, F. R. A. Estimate of Dose Distribution in Voxel Phantom Irradiated by a Planar Source Modeled by Translational Normal Probability Density Functions, 2012 International Symposium on radiation Physics, Rio de Janeiro, Brazil, 7-12 October, 2012.

13. XU, J. Practical WPF Charts and Graphics, APRESS, USA, 2009. 